COOLING SYSTEM

J. Marringer $1 / 27 / 86$ 
Pbar Note $4 / 51$ J. Marriner

January 27, 1986

\title{
NETWORK ANALYZER MEASUREMENTS FOR THE CORE MOMENTUM
}

\author{
COOLING SYSTEM
}

The purpose of this note is to describe a method for verifying the gain and phase response of the Accumulator core momentum cooling system using the network analyzer. is assumed that this method will be the primary method of adjusting the timing.

The formulation of the beam response to a longitudinal kicker has been given by van der Meer in CERN/PS/AA/80-4. His formula (rewritten by me) is:

$$
z_{b}^{-1}=\int c\left(f / f_{0}\right) P\left(f, f_{0}\right)\left(1+\frac{i}{\tan \left(\pi f / f_{0}\right)}\right)\left(\Psi+f_{0} \frac{d \Psi}{d f_{0}}\right) d f_{0}
$$

In this formula, $C$ is the factor that takes into account the different transit times due to differing revolution frequencies. The $P$ factor describes the pickup sensitivity - the $f_{0}$ dependence is the sensitivity of the pickup to different revolution frequencies (because the pickup may be in a region of dispersion) and the $f$ dependence is the sensitivity at the signal frequency (bandwidth, etc). As usual it is understood that one takes the principal value of the integral and one half of the pole term. The real part (absorptive) comes from the pole term and is proportional to the gradient of the distribution. Thus the resistance of the beam is positive or negative depending on which side of the peak in the frequency distribution is being observed. The imaginary part is capacitive at resonance (at the peak in the frequency distribution) and inductive far away. A plot of the beam response as measured by the network analyzer for a single schottky band is shown in figure 1 ab. With our technique of measuring the beam response, however, the gradient of the distribution is washed out a the frequency of the network analyzer signal. The net effect is 
that we only measure the reactive part of the beam response as is shown in figure 2ab. Probably the most important feature of this figure is the fact that the beam response at the center beam frequency is 900 - plus any phase shifts from the cooling system.

It is generally true that the beam response will show a $180^{\circ}$ phase shift (corresponding to negative feedback) if a system is properly phased for cooling. This is not the case for the system shown in figures 1 and 2 . These systems are assumed to be simple PU's and kickers which are not sensitive to momentum and there are no filters used. Under these circumstances there is no cooling and the phase at the peak of the distribution is 900 . If I recompute the beam response with the actual difference pickup used in the core cooling system, I get the response shown in figure 3ab where the $180^{\circ}$ phase at the center of the distribution is apparent. However, the gain at this point is ideally zero, making phase measurements somewhat tricky. Therefore, a slightly different procedure is recommended.

Good signal to noise ratios for these measurements can be obtained by placing the beam underneath either row of pickups in the difference array. One array is nominally at a frequency of $628.894 \mathrm{kHz}$ and the other at $6.28 .974 \mathrm{kHz}$. The exact values of the frequencies will vary with the setting of the bend bus and distortions of the closed orbit. The frequency difference, however, should be well determined. Figures $4 \mathrm{ab}$ and $5 \mathrm{ab}$ show the beam response when measuring a single schottky band with the beam underneath each of these pickups. Figures $6 \mathrm{ab}$ and $7 \mathrm{ab}$ are the same except they are measurements at the center frequency of every 10th schottky band from 2 to $4 \mathrm{GHz}$. These last displays are the ones which are most useful to time the system. The slopes of the phase vrs frequency should be equal and opposite if the delay is correct. The average value of the phase from the two sides should be $180^{\circ}$ otherwise a phase shifter is required. As for betatron cooling, the ideal amplitude response is flat with frequency. This corresponds to a linearly rising amplifier gain to compensate the linearly increasing width of the schottky bands. Figures 6 and 7 were generated assuming a gain independent of frequency. 


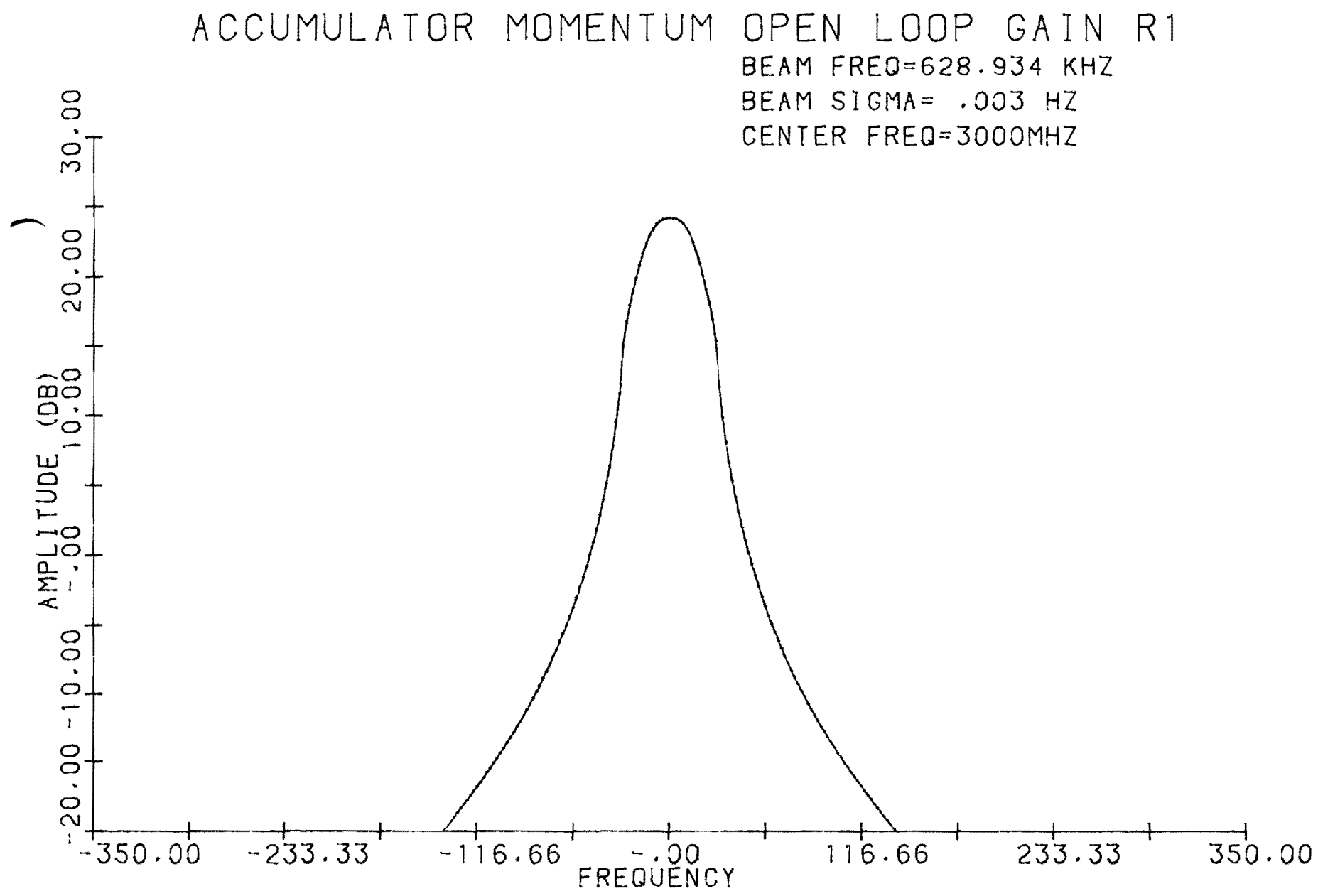

Figure 1 a 


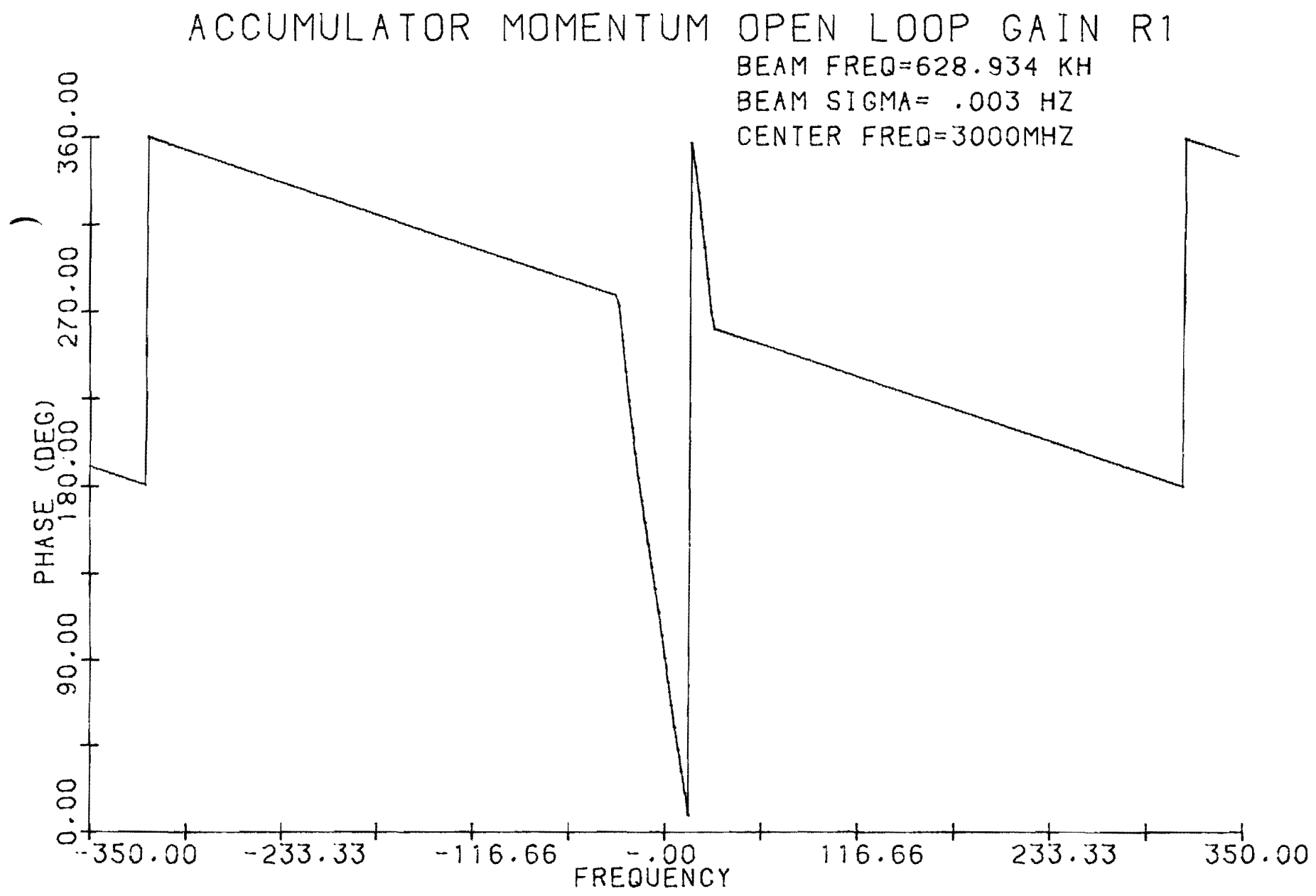

Figure $1 b$ 


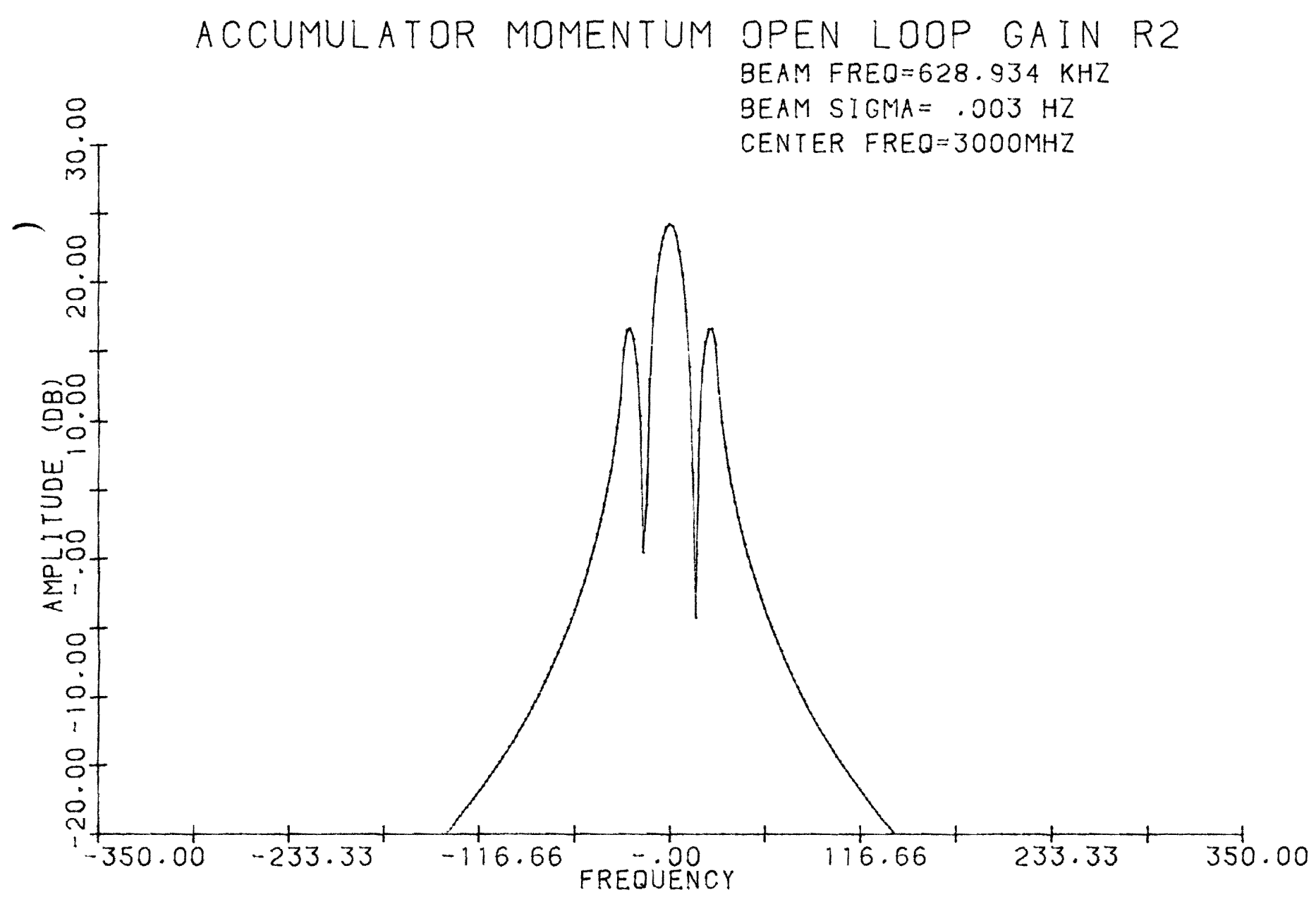

Figure $2 a$ 


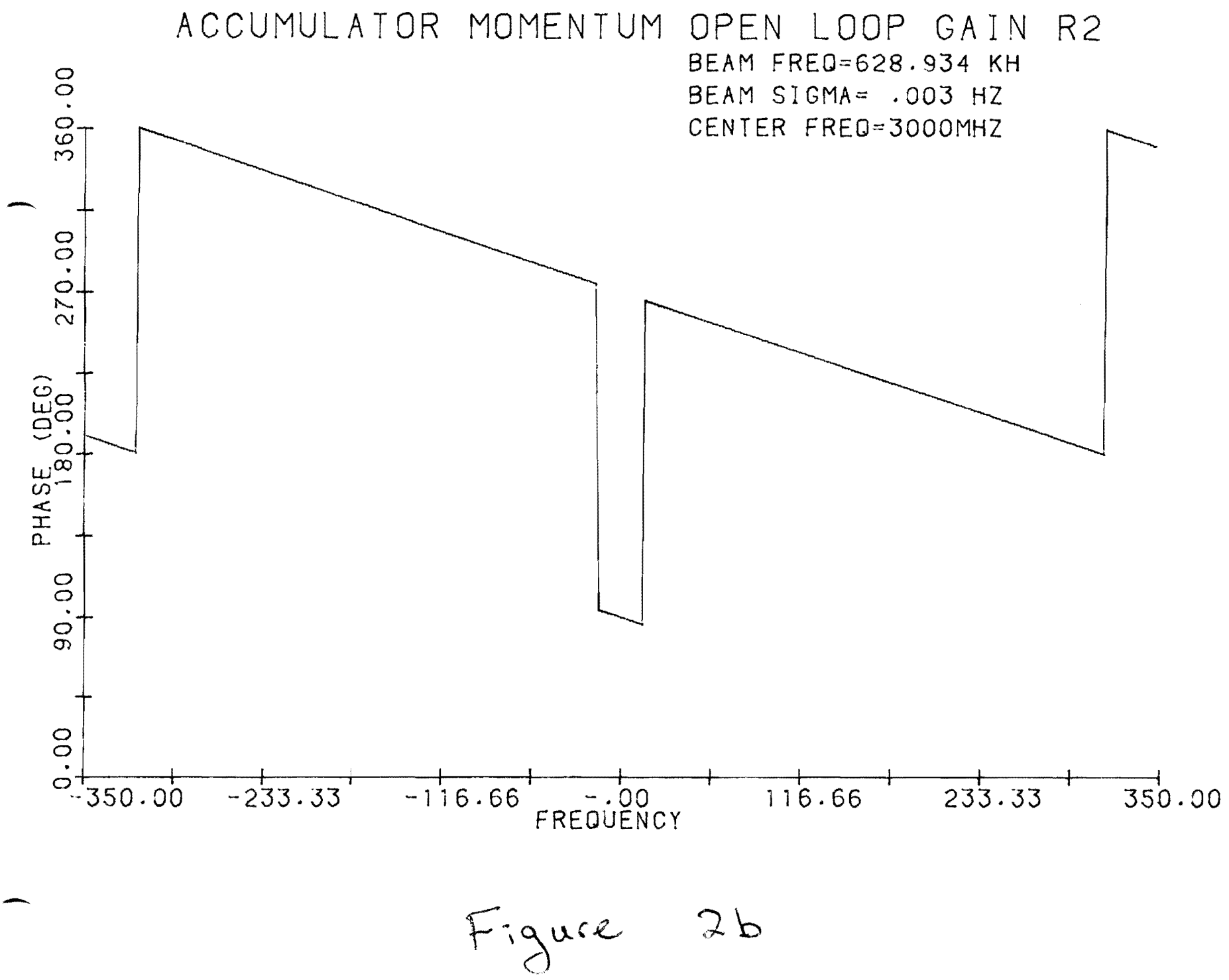




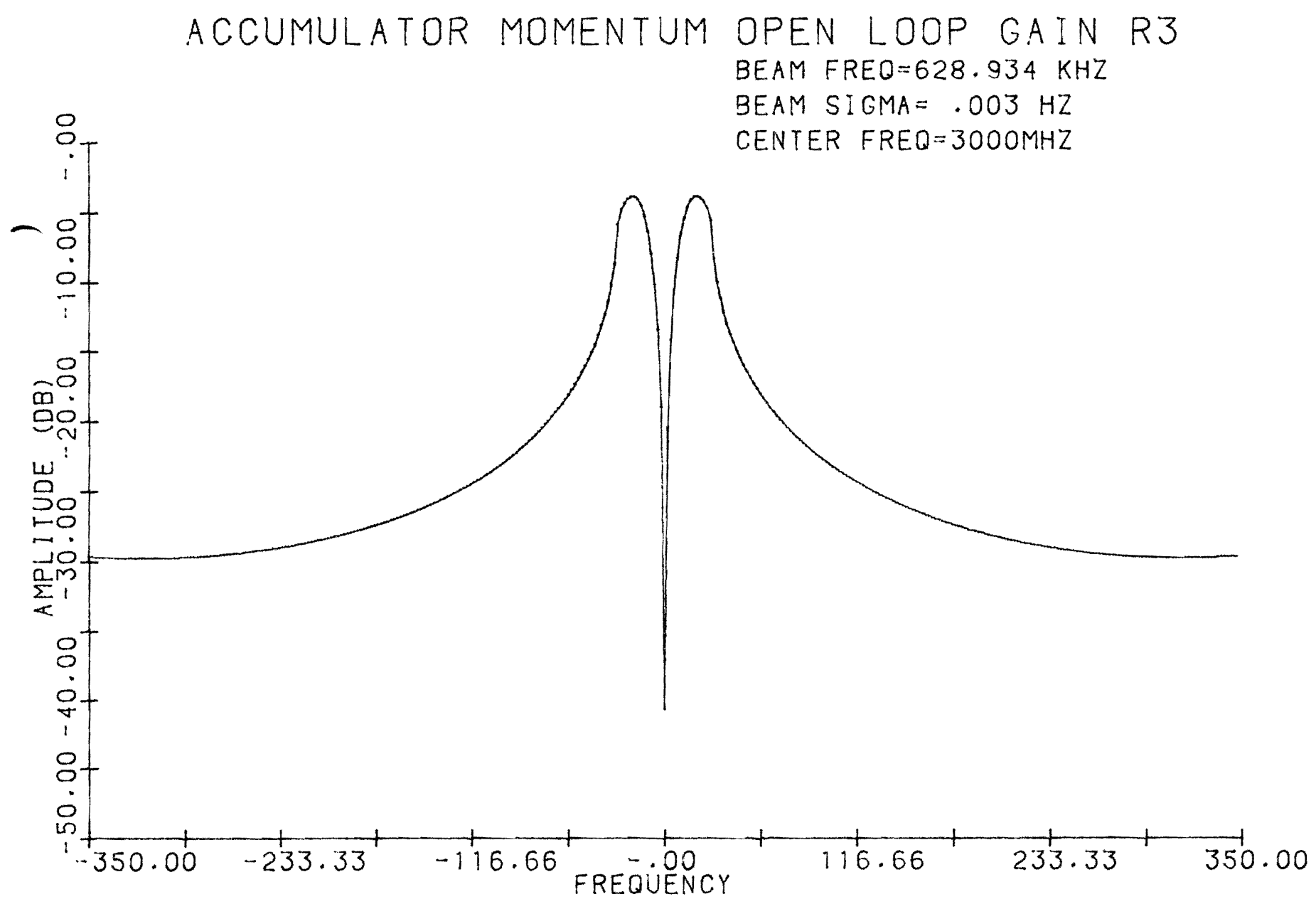

Figure $3 a$ 


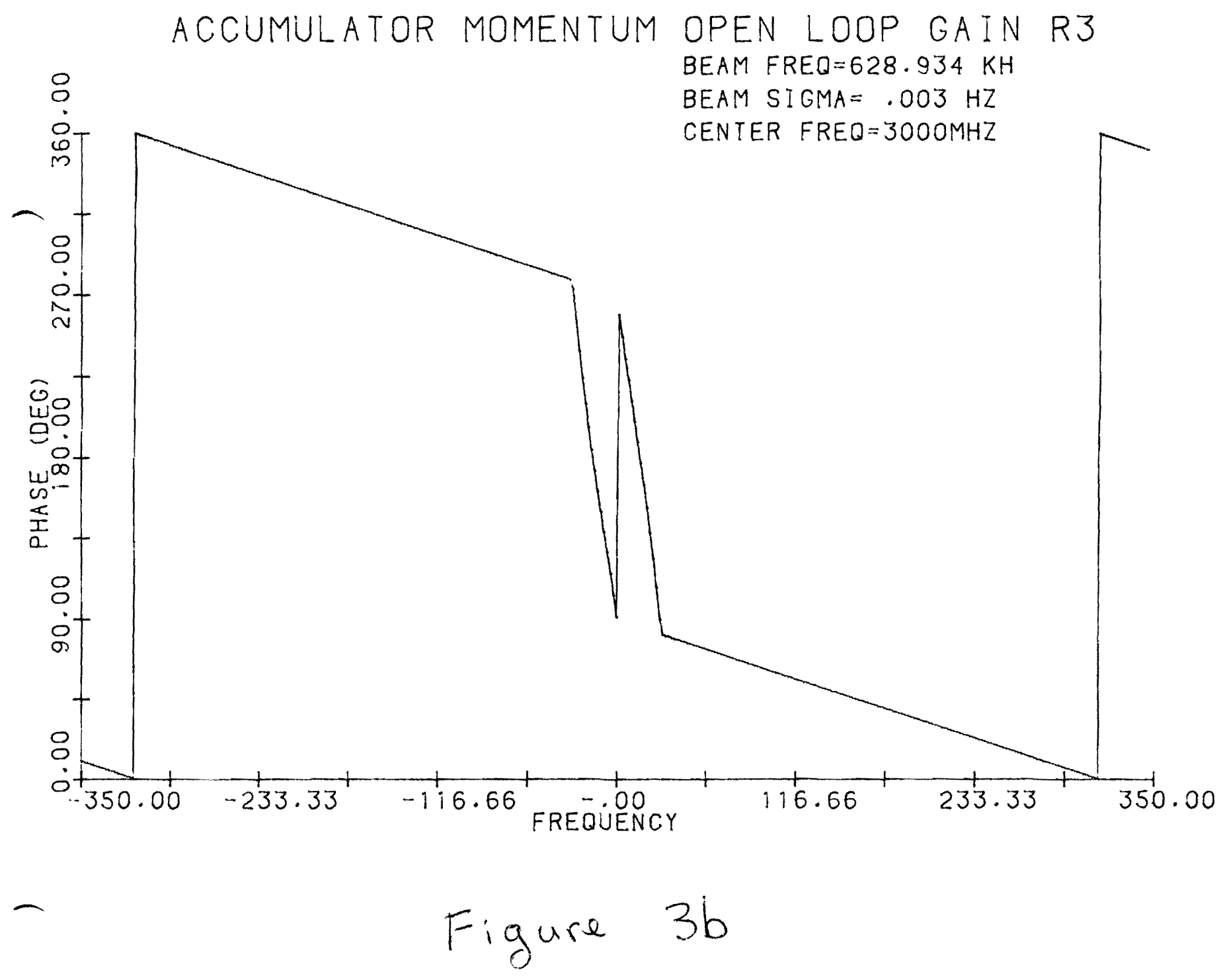




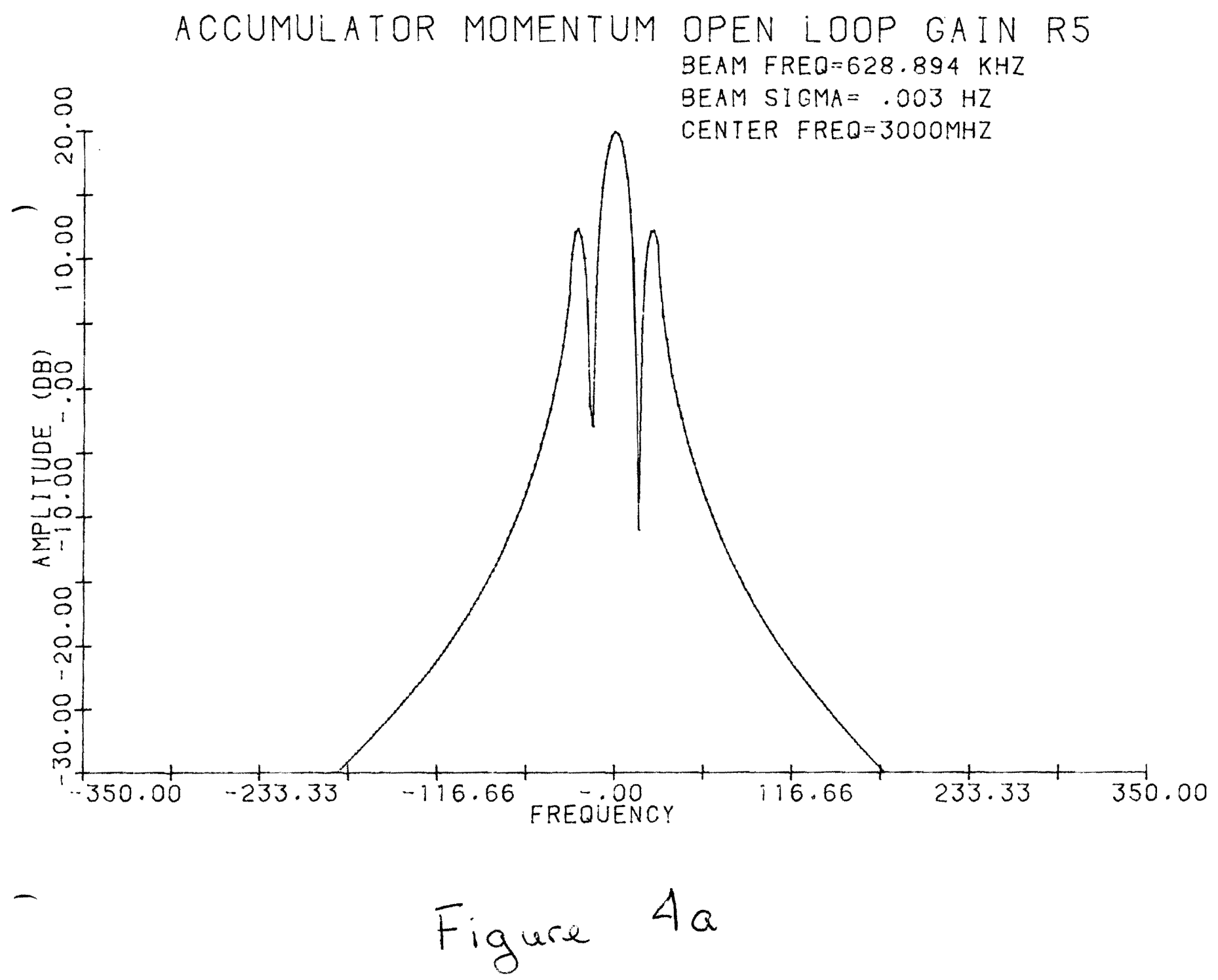




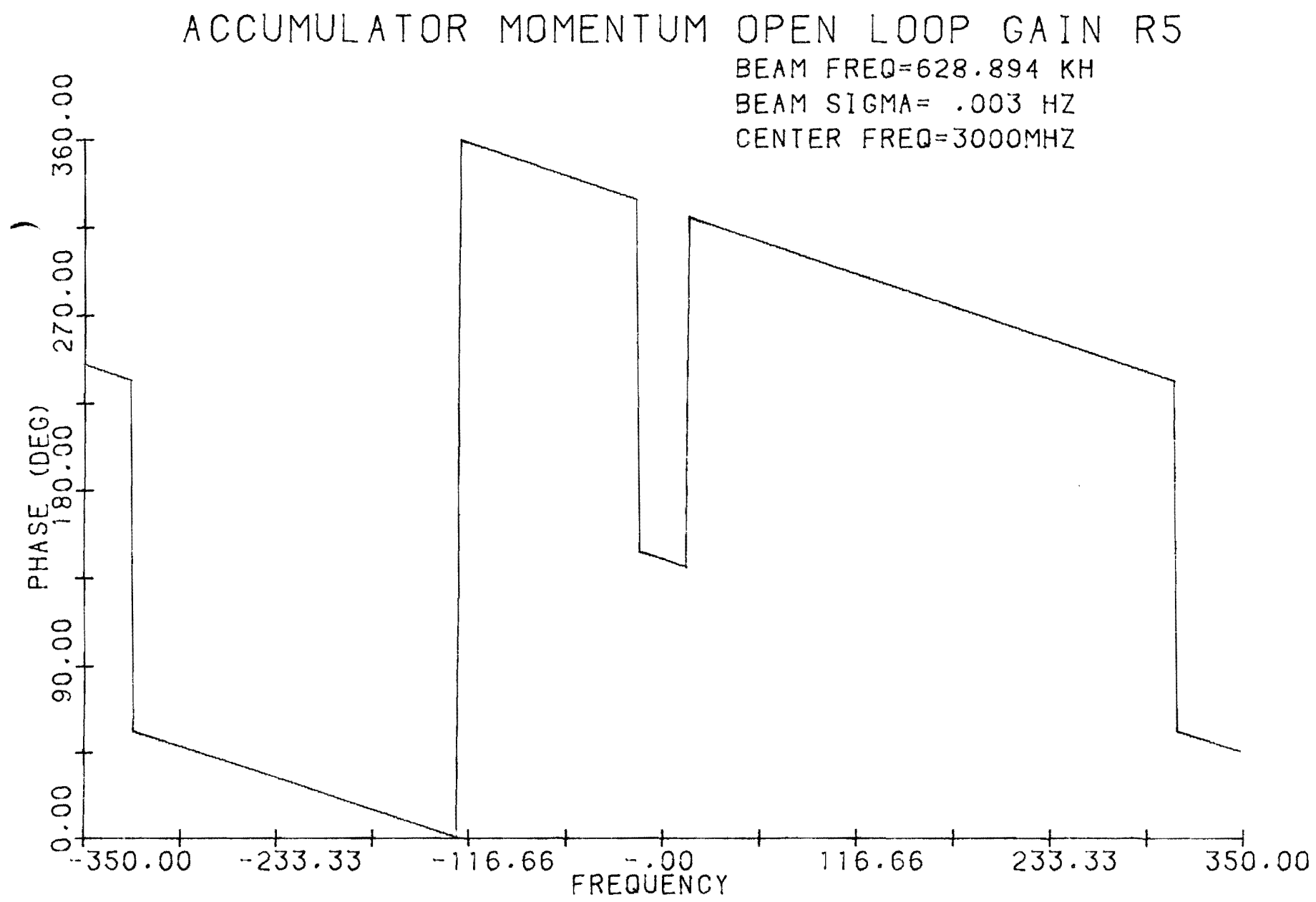

Figure $4 b$ 


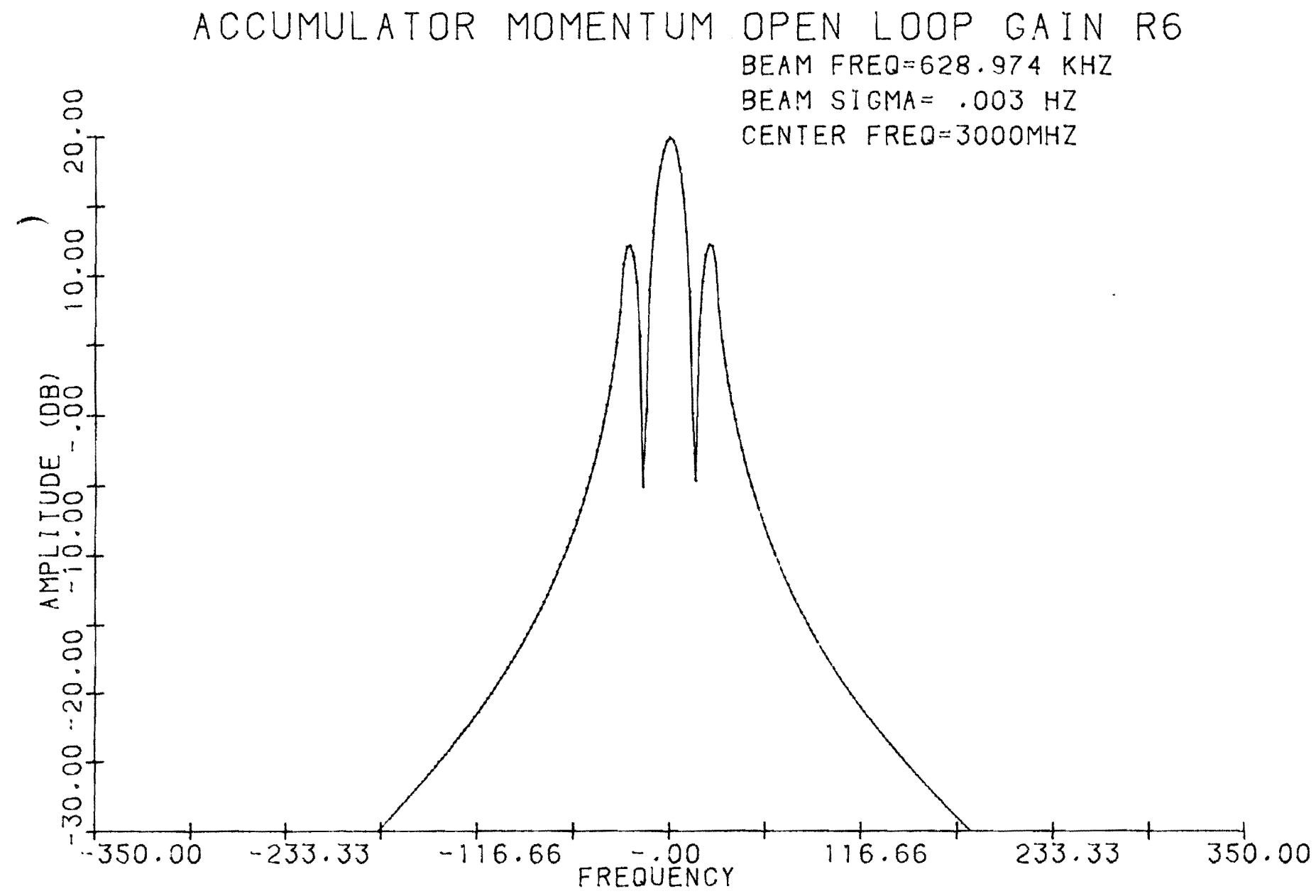

Figure $5 a$ 


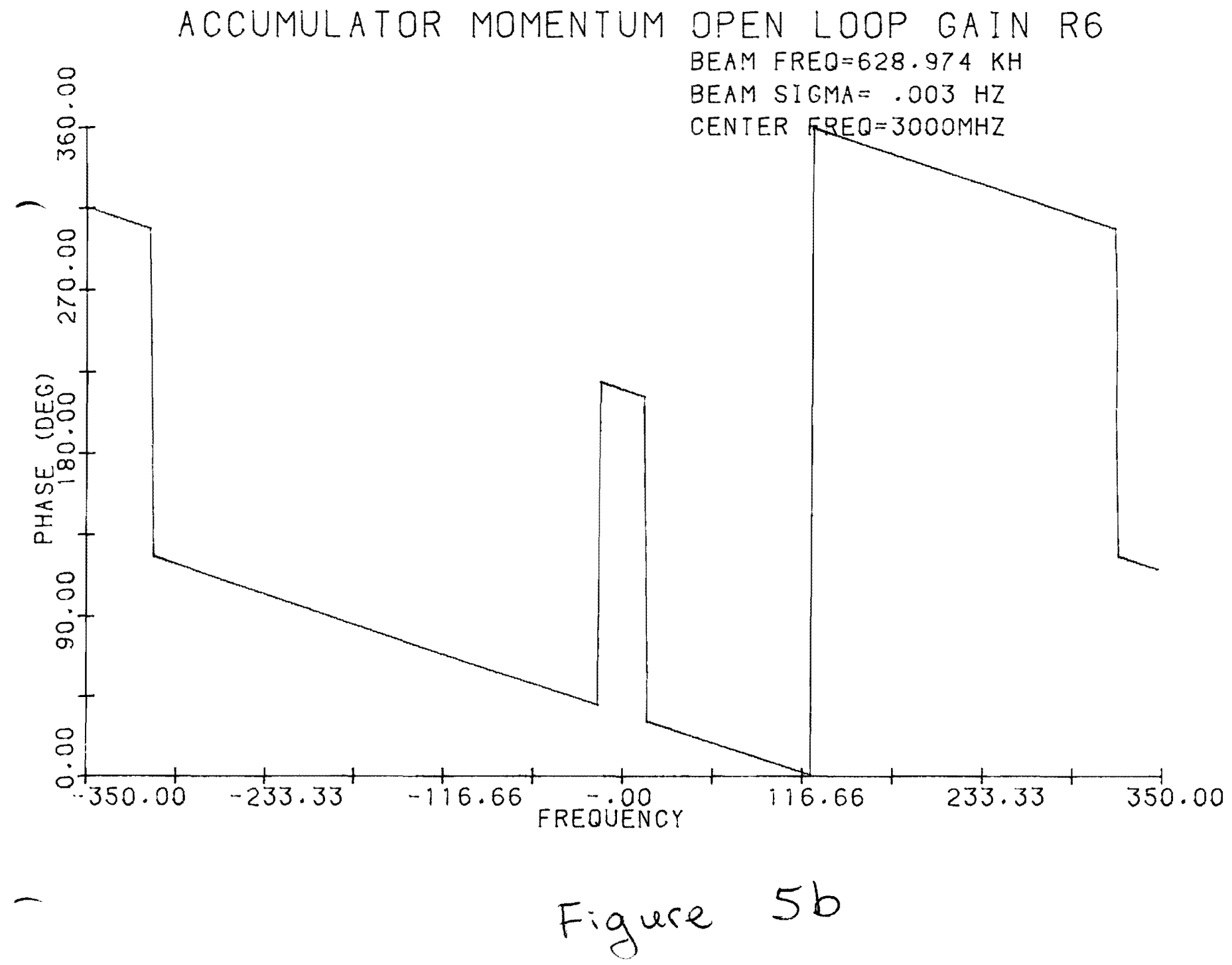




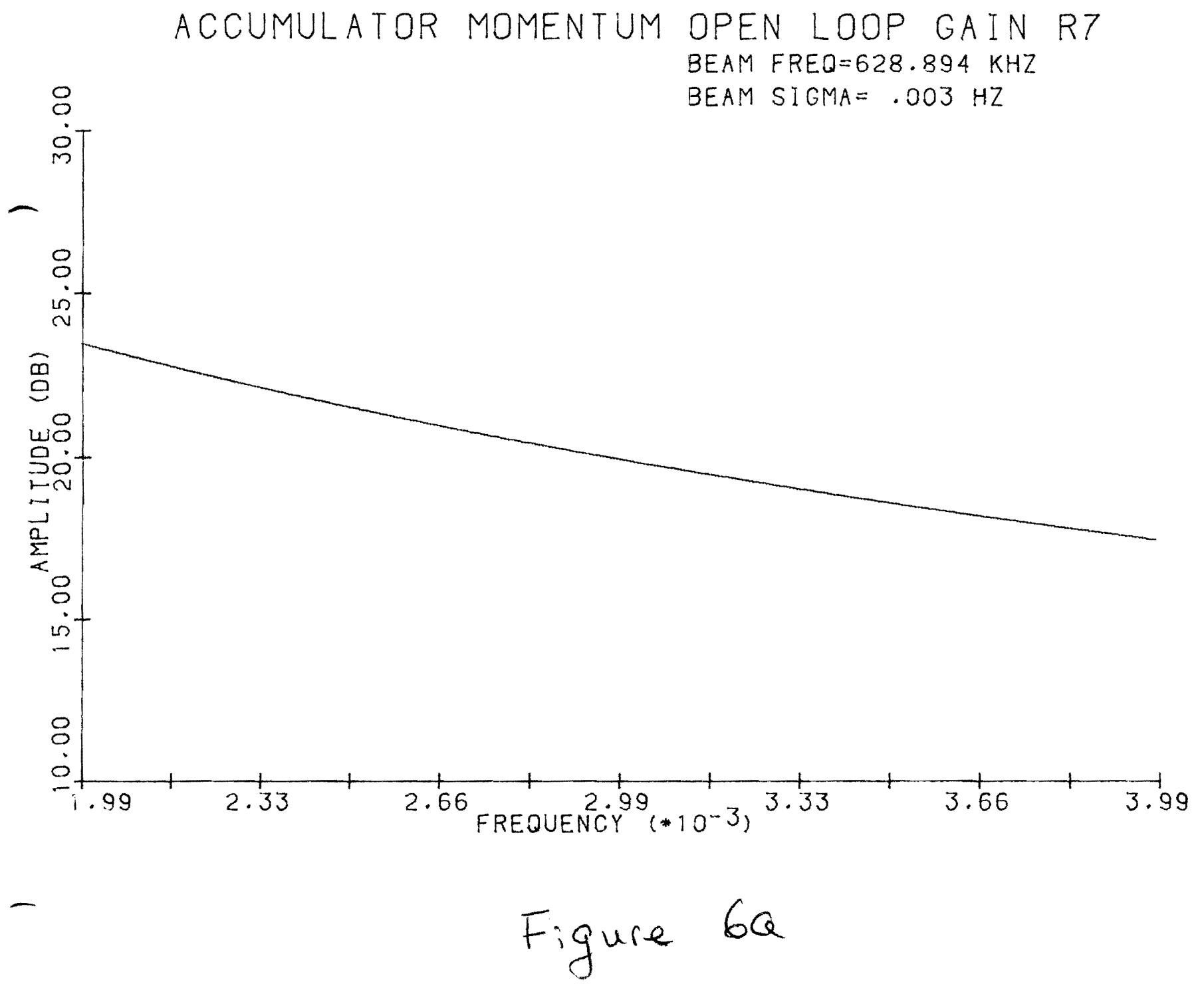




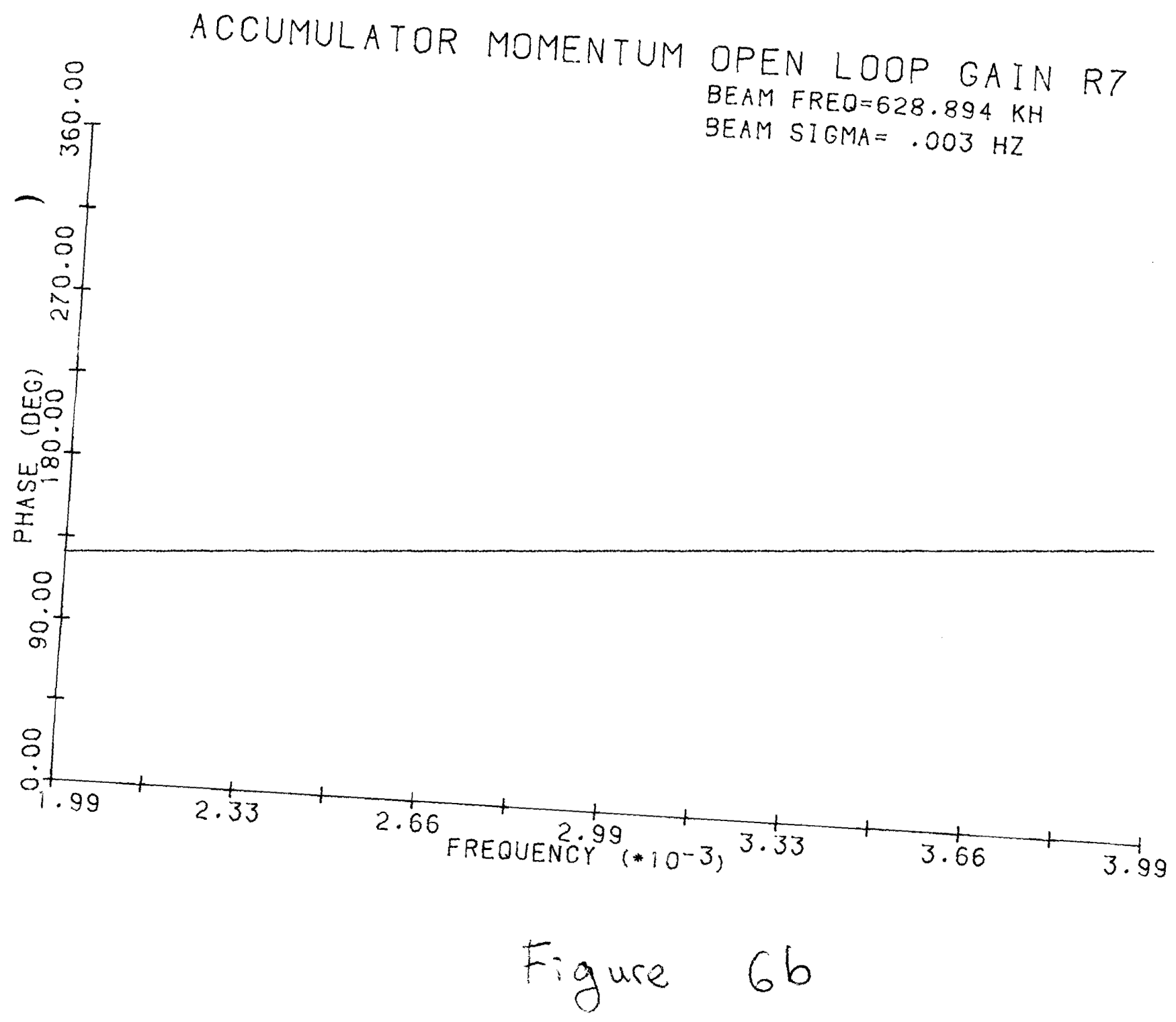




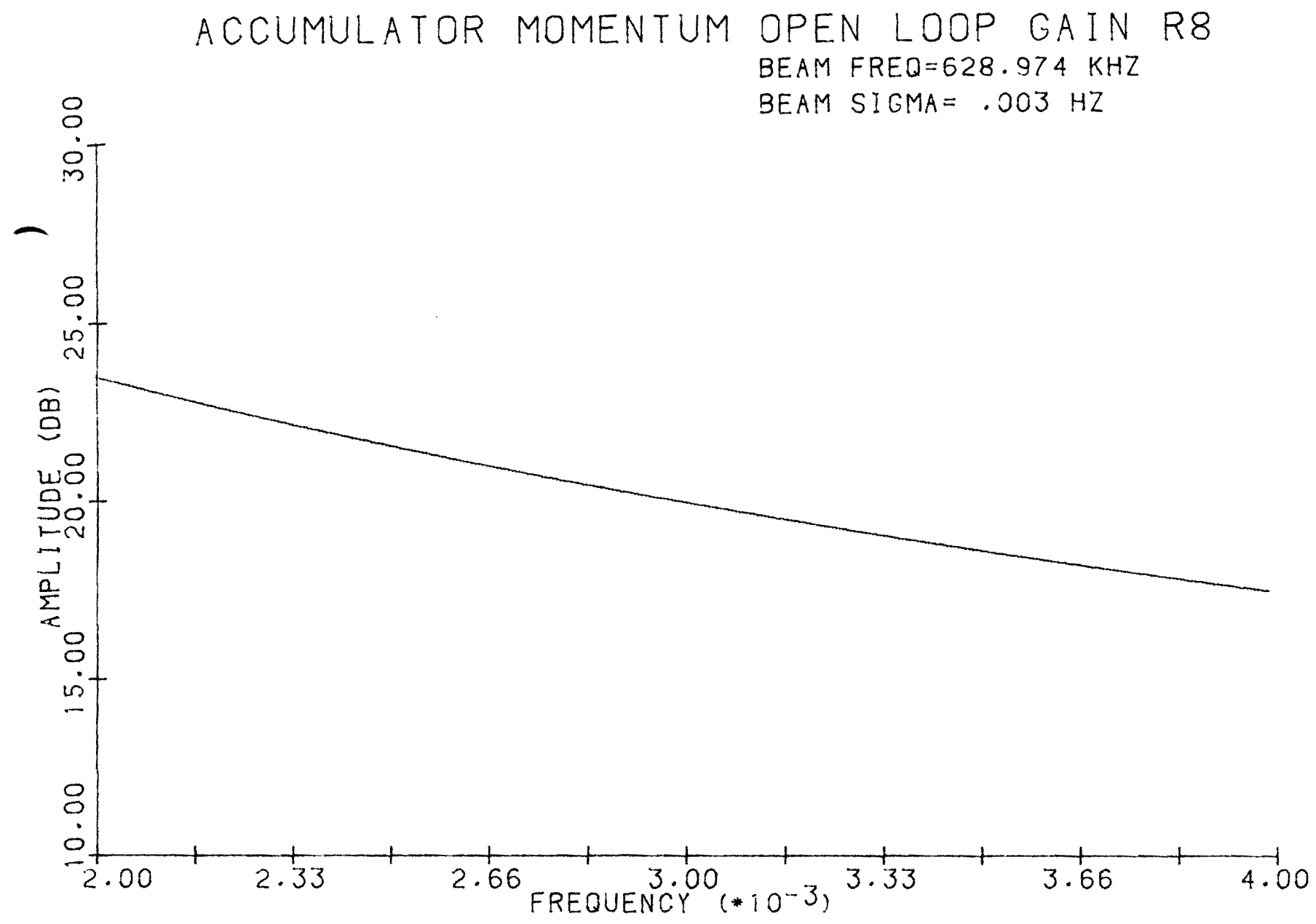

Figure $7 a$ 


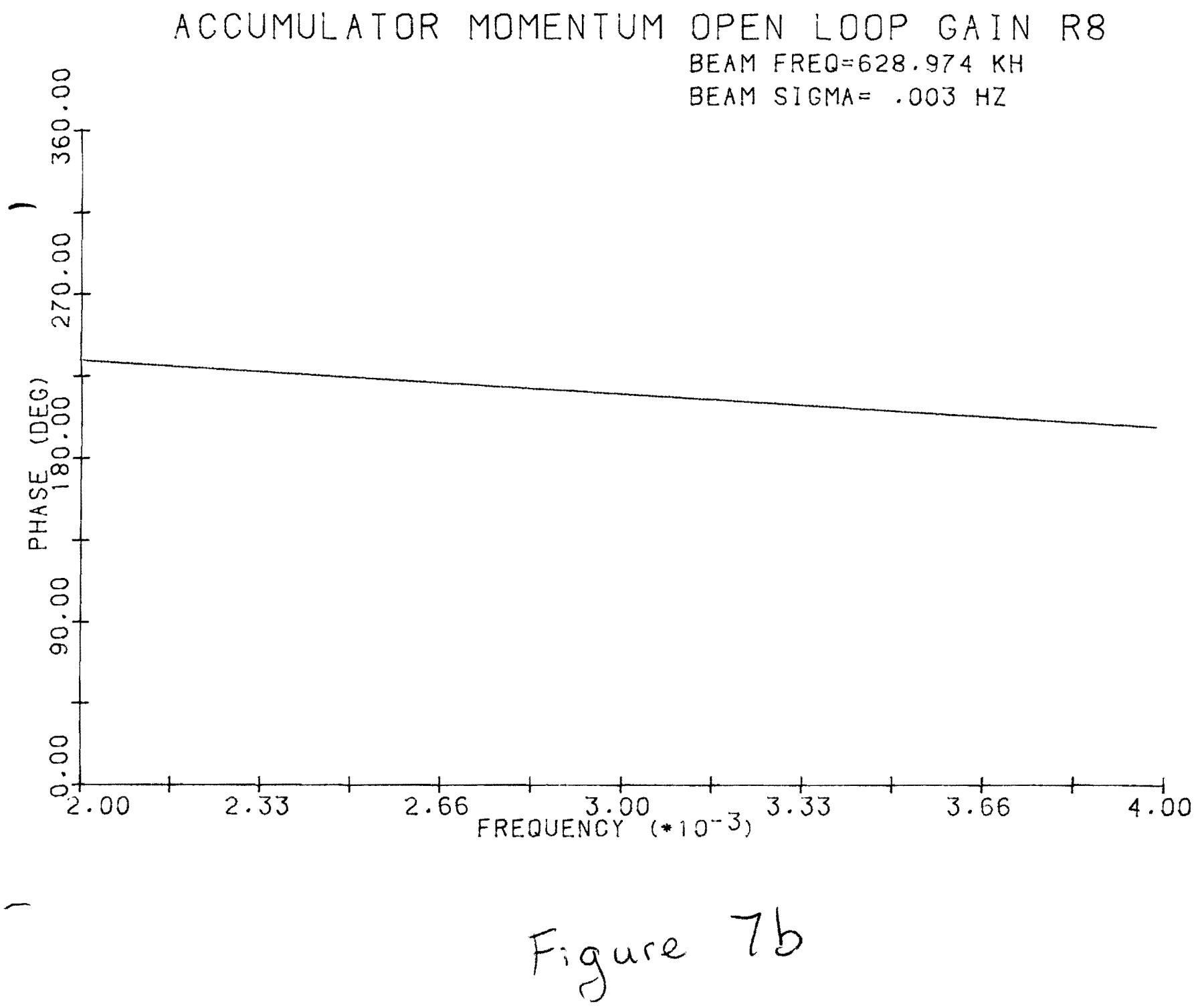

